\title{
Performance Analysis of VXLAN and NVGRE Tunneling Protocol on Virtual Network
}

\author{
Mirdan Syahid Mulya Sudrajat ${ }^{\star 1}$, Doan Perdana ${ }^{2}$, Ridha Muldina Negara ${ }^{3}$ \\ School of Electrical Engineering, Telkom University, Telekomunikasi street no.1, Bandung, \\ West Java, Indonesia \\ ${ }^{*}$ Corresponding author, e-mail: mirdsmulya@gmail.com ${ }^{1}$, doanperdana@telkomuniversity.ac.id ${ }^{2}$, \\ ridhanegara@telkomuniversity.ac.id ${ }^{3}$
}

\begin{abstract}
Virtualization is a new revolutionary approach in networking industry, its make possible to build several virtual machine (VM) in one physical hardware. In virtualization practice, one VM might be connected to others, but not all of VM in one environment must be connected due the privacy and security issues. One of the solutions which can address this issue is tunneling protocol. Tunneling protocol is a layer-2-in-layer-3 protocol which can isolate tenant traffic in virtualize environment. This research conducted about the performance of VXLAN and NVGRE tunneling protocol which works on virtualize environment and aims to determine the perfomances of throughput, delay, jitter, and vCPU Usage using variable packet size in range of 128-1514 byte. From the the result, can be conclude that both of tunneling protocol can isolate the traffic between tenant. For the performance result, NVGRE has the highest value of throughput, 771,02 Mbps and the VXLAN got 753,62 Mbps. For the delay NVGRE got $2.24 \mathrm{~ms}$ and VXLAN got $2.29 \mathrm{~ms}$. For the jitter, NVGRE has better rate value of $0.361 \mathrm{~ms}$, than VXLAN value of 0.348 $m s$, and the VCPU usage performance, NVGRE has the highest performance too that value is $60.57 \%$. So on overall performance NVGRE has the better performance than VXLAN.
\end{abstract}

Keywords: tunneling protocol, network virtualization, overlay network

\section{Introduction}

In this era of global competition, the availability of cloud computing infrastructure in a corporate or agency environment becomes very important. In practice, there are often occurs problems faced. Those problems are the scalability and flexibility of the cloud computing infrastructure [1]. As the massive ICT development, one the emerging solution to simplify these problems is approaching virtualization technology. Which has been widely implemented by small and large scale ICT companies like datacenter [1]. This virtualization technology can't be denied that can decreasing the CAPEX and OPEX side [2].

Virtualization is the use of software to allocate the resources of hardware into several parts like virtual machine (VM). With virtualization, the network complexity can be simplified, and can be deployed according to user needs flexibly and scalable [3]. In datacenter-scale virtualization, which can accommodate a large number of tenants. Each tenant may be has more than one integrated VM to execute an application, so each tenant must be isolated from others [4]. One of the solution that arises to address those problems is a tunneling protocol technology. This protocol is able to isolate traffic within the virtualization technology which called overlay virtual network (OVN) [5]. There are also another way that use of VLAN technology (802.1Q). However, this technology is considered that not reliable enough because of scalability issue. VLAN relatively low if its compare with the tunneling protocol technology, because VLAN only has the capacity of VLAN tag around 4000 VLAN ID.

Tunneling protocols that works in virtual environments or called with overlay virtual network, has three protocols, namely VXLAN, NVGRE, and STT [6]. These tunneling protocols can isolate tenant traffic up to 16 million tunnel ids [6], coupled with a multitenancy features that increase tenant flexibility. But in implementation of this technology there are some shortcoming issues, which can burden of VCPU usage and bandwidth effectiveness, these because the addition of encapsulation process to the packet before its transmitted, so the vCPU usage needs to work more than before, along with the increasing the packet size as well [6]. At this 
research will analyze the performance comparison of the two tunneling protocols, there are NVGRE and VXLAN. It aims to see which one has the better performance.

\section{Research Method}

This research used system architecture as shown in Figure 1. and the topology shown in Figure 2. which consists of two servers, user PC and one switch. Each server is built in a virtual environment using the Xen Server hypervisor and thereafter built one VM from Ubuntu Linux as host. User PC is used as hypervisor manager by installing Xen Center which is the default application of Xen Hypervisor that serves to control and monitoring virtual resources remotely. Once the system is ready, the tunneling protocol is built using bash scripting on each VM that has previously been installed Open vSwitch. Open vSwitch is use as a tunnel end point to implementing this tunneling protocol at the system [7].



Figure 1. Tunneling system architecture

There are four test scenarios performed to see the performance of the tunneling protocol VXLAN and NVGRE, which work in virtual environment [8]. In addition, the two of tunneling protocols were also tested native-based communication (non-tunneling), as a comparison with the two tunneling protocols tested. There are 4 parameters that tested in these experiments, those are throughput testing, delay, jitter, and vCPU Usage [9]. This test is based on the size of the packet size transmitted by VM A as the sender, to the VM B as the receiver, which is $64,128,256,384,512,640,768,896,1024,1153,1280,1408$, and 1514 bytes [9].

These metric value were measured by using D-ITG application. The D-ITG has capable to producing traffic with various packet level. D-ITG can be used to measure throughput, delay, jitter across these virtual environment system [9-11]. To ensure the data accuracy, all the test were execute 20 times, and each run had duration of 10 second [12-13]. Table 1 show virtual machine specification

Table 1. Virtual Machine Specification

\begin{tabular}{ccccccc}
\hline Location & Guest & OS & vCPU & RAM & Hypervisor & vSwitch \\
\hline Server A & VM A & Ubuntu & \multirow{2}{*}{ 1 Core } & \multirow{2}{*}{ 1 GByte } & XenServer & Open vSwitch \\
& & 16.04 & & & 7.0 & 2.5 .1 \\
Server B & VM B & Ubuntu & \multirow{2}{*}{ 1 Core } & 1 GByte & XenServer & Open vSwitch \\
& & 16.04 & & & 7.0 & 2.5 .1 \\
\hline
\end{tabular}






Figure 2. Tunneling system architecture

\section{Results and Analysis}

The test is conducted to find out which tunneling protocol has the most optimal throughput value and measure how far the trade-off occurs between VXLAN and NVGRE and also with native. Based on Figure 3, it can be seen that there is difference of throughput value between VXLAN and NVGRE performance. The difference occurs when the size of the tested packet size is $128-1514$ bytes. NVGRE performance has a greater throughput value than VXLAN performance throughout the packet size testing is increased.

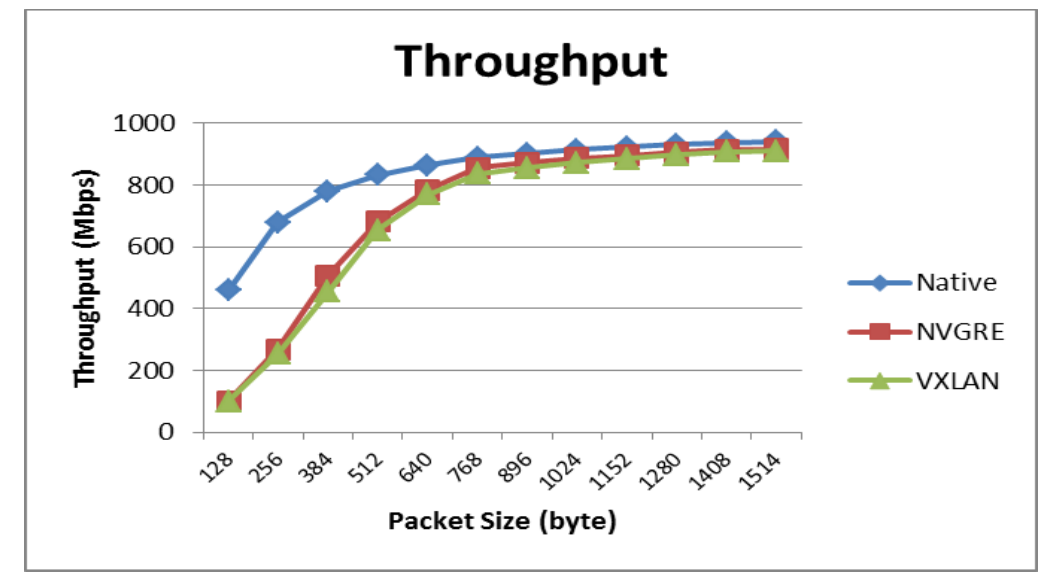

Figure 3. Throughput performance from VM-A to VM-B

The test is conducted to find out which tunneling protocol has the most optimal throughput value and measure how far the tradeoff occurs between VXLAN and NVGRE and also with native. Based on Figure 3, it can be seen that there is difference of throughput value between VXLAN and NVGRE performance. The difference occurs when the size of the tested packet size is $256-1514$ bytes. NVGRE performance has a greater throughput value than VXLAN performance throughout the packet size testing is increased. These performance distance is due the different characteristics of the number of packet header use for each tunneling platform, the NVGRE has 4 byte GRE header, and a 16 byte VXLAN protocol consisting of 8 bytes of VXLAN headers and 8 bytes of user datagram protocol (UDP). With less 
packet headers, its proven that NVGRE have higher throughput performance than VXLAN. For the most optimal packet size performance, both of tunneling protocol has the same variable which is at 1514 bytes, with VXLAN throughput performance of $908.55 \mathrm{Mbps}$ and NVGRE of $916.60 \mathrm{Mbps}$. It caused of the packet size can impact on the utility of bandwidth usage, so when the packet size is 1534 bytes, each protocol gets the most optimal throughput.

In Figure 4 shows that the result has the different delay performance. Each tunneling protocol has a tendency to increase delay value when packet size is tested larger. Tunneling protocol that has the highest delay performance is VXLAN, it takes on packet size above 768 bytes with delay value $2.98 \mathrm{~ms}$. The maximum performance of delay is not exceeding $3 \mathrm{~ms}$, so the delay performance in tunneling protocol on virtualization environment is very reliable to use, because the delay performance accepted with ITU G.1010 standard, related to server access, which is has $<2$ s standard. This also shows that the performance of Open vSwitch as a tunnel end point is able to work efficiently, and the delay performance is not too far away from native performance. Figure. 5 shows Jitter performance from VM-A to VM-B.

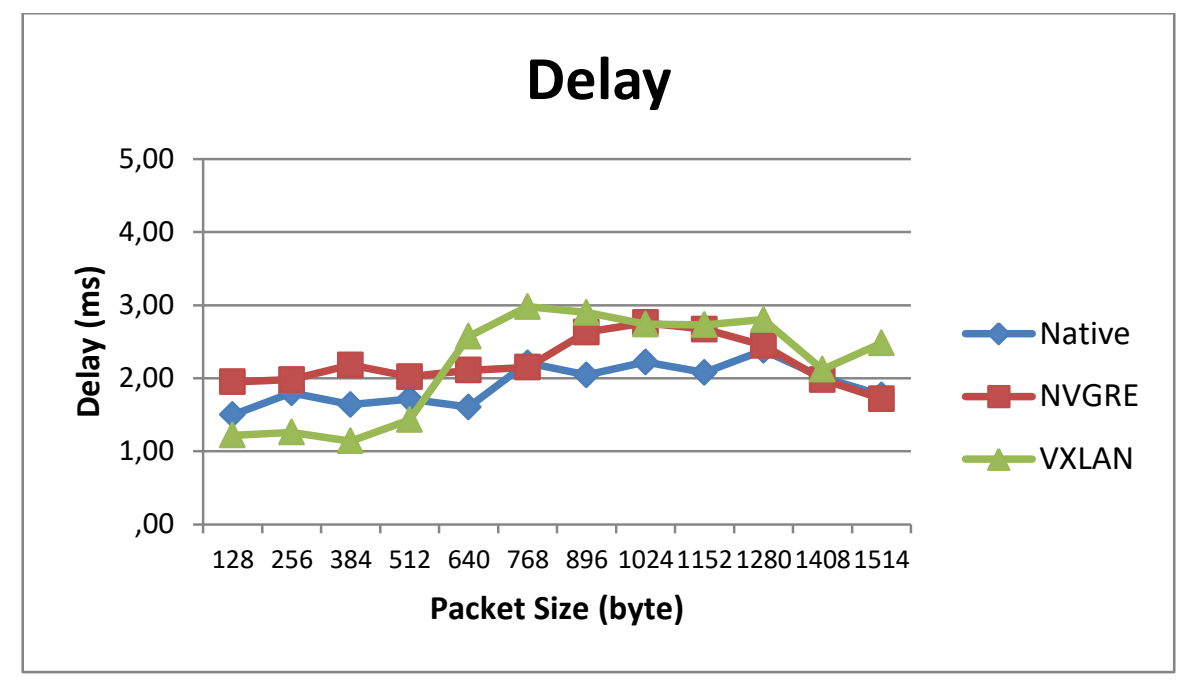

Figure. 4. Delay performances from VM-A to VM-B

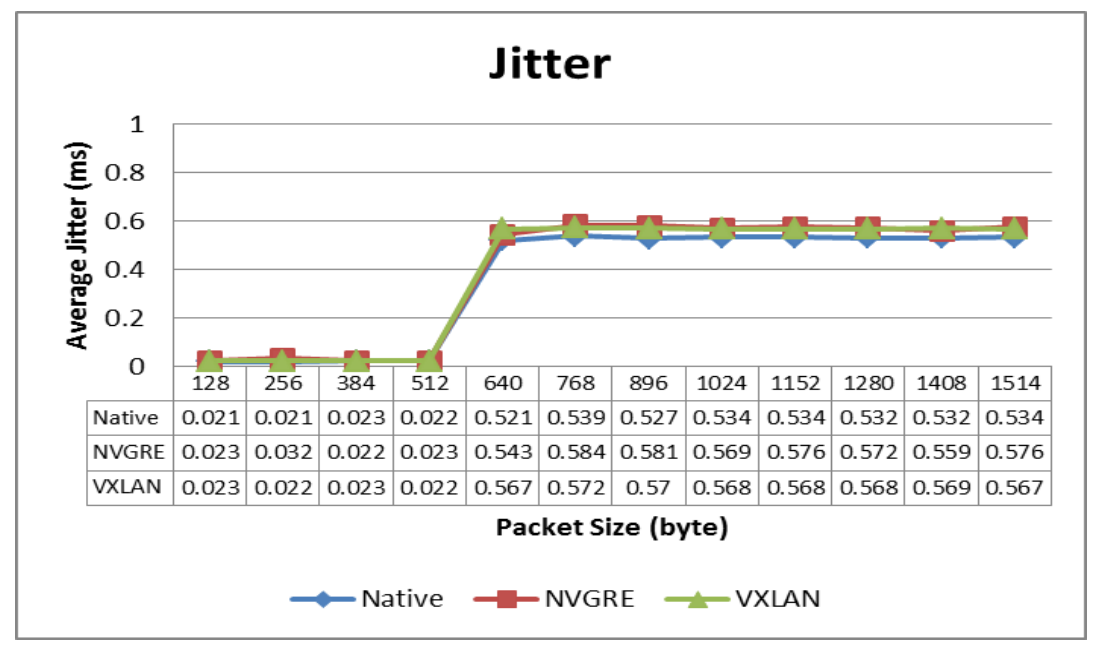

Figure. 5. Jitter performance from VM-A to VM-B

There are two sides of the test of vCPU usage performance, at the sender (VM-A) and recipient side (VM-B). Testing is conducted by sending traffic from VM-A to VM-B by using a 
different variable packet size as shown in Figure 6. For the test results at the sender can be seen in Figure 6 . From the images shown that the VXLAN protocol requires the largest vCPU usage compared to NVGRE and Native performance. The performance of VXLAN protocol with NVGRE has the same trend of test result, that is the bigger packet size used then the vCPU usage performance will decrease. This is due to the greater packet size used, will reduce the ratio of the number of packet deliveries per second to the bandwidth, so the number of packets sent per second will decrease.

With the decreasing number of packets per second transmitted, it will be directly decline the number of packets that must be encapsulated, so the performance of vCPU usage decreases. At the test result on VM-B can be seen in Figure 7, from the graphic image shows the vCPU usage in VM-B as a whole has increased performance value compared to VM-A, this performance improvement shows that $v C P U$ usage during the process of decapsulation in receiver (VM-B), requires more $\mathrm{VCPU}$ usage than the sender's encapsulation process.

\section{vCPU Usage VM A}

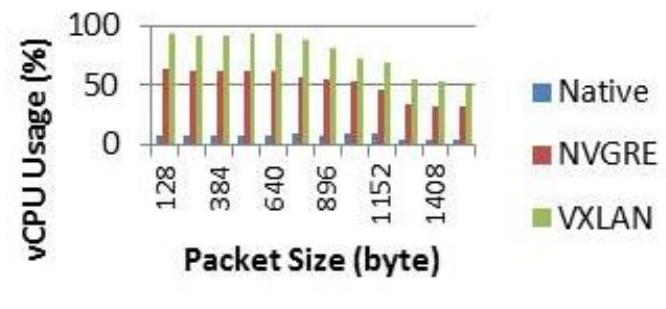

Figure 6. vCPU Usage VM-A

\section{vCPU Usage VM B}

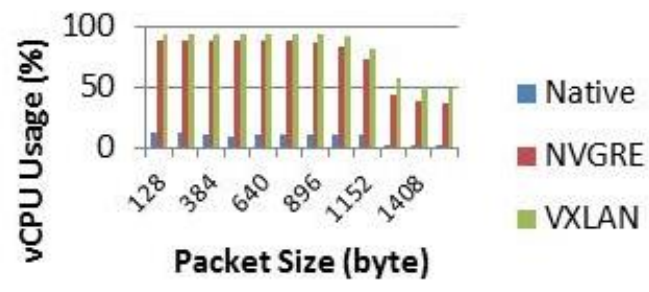

Figure 7. vCPU Usage VM-B

\section{Conclusion}

We conclude from the test results and performance analysis of tunneling protocol it shows that the tunneling protocol, VXLAN and NVGRE has the different performances. The best tunneling protocol in terms of bandwidth usage efficiency is the NVGRE tunneling protocol, with an average use of throughput is $771,02 \mathrm{Mbps}$ while VXLAN has 753,62 Mbps. Then the delay test shows that the tunneling protocol which has the lowest average travel time is the NVGRE tunneling protocol, with the average delay value of $2.24 \mathrm{~ms}$, while the VXLAN is $2.29 \mathrm{~ms}$. The second, delay performance of this protocol is in accordance with ITU.TG.1010 related on server access is <2s. In jitter performance shows that the tunneling protocol which has the lowest variance delay rate is the VXLAN tunneling protocol with jitter value is $0.348 \mathrm{~ms}$ while NVGRE performance is $0.361 \mathrm{~ms}$. The last is the CPU Usage performance shows that the tunneling protocol which has the lowest of VCPU usage is the NVGRE tunneling protocol, with the average CPU Usage value on the sender and recipient host is $60.57 \%$ and the VXLAN performance is $79.84 \%$.

For overall, it can be concluded that the most optimal tunneling protocol performance is NVGRE because its superiority from 3-of-4 parameters, there are the throughput, vCPU usage, and delay performance.

\section{References}

[1] A Leen, H Franke, C Li, W Liao. Toward Performance Optimization with CPU Offloading for Virtualized Multi-Tenant Data Center Networks. In IEEE 6th International Conference on Cloud Computing Technology and Science, 2016.

[2] R Kawashima, H Matsuo. Implementation and Performance Analysis of STT Tunneling using VNIC Offloading Framework (CVSW). In IEEE 6th International Conference on Cloud Computing Technology and Science. 2014.s

[3] L Lewin-Eytan, K Barabash, R Cohen, V Jain, A Levin. Designing Modular Overlay Solutions for Network Virtualization. In IBM Research Report. 2011. 
[4] M Sridharanm K Duda, I Ganga, A Greenberg, G Lin, M Pearson, P Thaler, C Tumuluri, N Venkataramiah, Y Wang. NVGRE: Network Virtualization using Generic Routing Encapsulation. 2011.

[5] Larry L Peterson, Bruce S Davie, Computer Networks, Fifth Edition. Burlington: Elsevier.Inc. 2012

[6] Nadeem, M Aamir Karamat, Taimur. A Survey of Cloud Network Overlay Protocols. In IEEE Sixth International Conference on Digital Information and Communication Technology and its Applications (DICTAP), 2016.

[7] Piccolo, V Dell Amamou, Ahmed. Haddadou, Kamel, Pujolle, Guy. A Survey of Network Isolation Solutions for Multi-tenant data center. In IEEE Communication Survey \& Tuttorials. 2015.

[8] Narayan Shaneel, Tauch Sotharith. Network Performance Evaluation of IPv4-v6 Configured Tunnel and 6to4 Transition Mechanism on Windows Server Operating Systems. In IEEE International Conference on Computer Design and Applications (ICCDA). 2010.

[9] Alessio Botta, Alberto Dainotti, Antonio Pescape. A tool for the generation of realistic network workload for emerging networking scenarios. Journal Computer Networks: The International Journal of Computer and Telecommunications Networking. 2012; 56(15): 3531-3547.

[10] R Kawashima, Ryota Matsuo. Non-Tunneling Edge-Overlay Model Using OpenFlow for Cloud Datacenter Networks. In IEEE International Conference on Cloud Computing Technology and Science. 2013.

[11] Perdana, Doan, Riri Fitri Sari. Multicast Routing Performance Analysis for Mobility Models on the IEEE 1609.4 Standard using Random Dijkstra Algorithm. The 1st International Conference on Intelligent Green Building and Smart Grid (IGBSG 2014), Taiwan, April 2014.

[12] Munadi, Rendy, et al. Adaptive Control Channel Interval in VANET Based on Mobility Model and Queuing Network Analysis. Journal of Networks. 2016; 11(2): 62-68. Academic OneFile, Accessed 12 July 2017.

[13] Perdana, Doan, Riri Fitri Sari. Enhancing Channel Coordination Scheme Caused by Corrupted Nakagami Signal and Mobility Models on the IEEE 1609.4 Standard. JNW 9.12 (2014): 3454-3461. 